Motrivivência Ano XXIV, No 38, P. 89-97 Jun./2012

\title{
MEMÓRIA E PROGRAMAS SOCIAIS DE ESPORTE E LAZER: o acervo do Programa Segundo Tempo do Repositório Digital do Centro de Memória do Esporte
}

\author{
Silvana Vilodre Goellner \\ Naila Touguinho Lomando² \\ Ivone $\mathrm{Jab}^{3}$ \\ Luciane Silveira Soares
}

\section{RESUMO}

Considerando a importância da produção e difusão de conhecimentos, este texto tem como objetivo apresentar algumas atividades desenvolvidas pelo Centro de Memória do Esporte da Escola de Educação Física da Universidade Federal do Rio Grande do Sul (CEME) no que tange à preservação da memória do esporte e do lazer. Para tanto centraliza sua abordagem na recente criação de seu repositório institucional, o qual abriga o acervo Memória do Programa Segundo Tempo, cujo objetivo é criar estratégias de divulgação da produção deste programa social de esporte e lazer de forma a preservar sua memória e divulgá-la à comunidade em geral.

Palavras-chave: memória; esporte; projetos sociais.

1 Professora do Departamento de Educação Física da UFRGS. Coordenadora do Centro de Memória do Esporte. Pesquisadora do CNPq. Contato: vilodre@gmail.com.

2 Bibliotecária da Escola de Educação Física da UFRGS. Contato: nailalomando@yahoo.com.br.

3 Bibliotecária da Escola de Educação Física da UFRGS. Doutorando do Programa de Pós-Graduação em Ciências do Movimento Humano - UFRGS. Contato: ivonejob@yahoo.com.br

4 Bibliotecária. Mestranda do Programa de Pós-Graduação em Ciências do Movimento Humano - UFRGS. Contato: luzbib@gmail.com 


\section{INTRODUÇÃO}

Considerando a importância da produção e difusão de conhecimentos, este texto tem como objetivo apresentar algumas atividades desenvolvidas pelo Centro de Memória do Esporte da Escola de Educação Física da Universidade Federal do Rio Grande do Sul (CEME) no que tange à preservação da memória do esporte e do lazer. Para tanto centraliza sua abordagem na recente criação de seu repositório institucional, o qual abriga o acervo Memória do Programa Segundo Tempo, cujo objetivo é criar estratégias de divulgação da produção deste programa social de esporte e lazer de forma a preservar sua memória e divulgá-la à comunidade em geral.

Criado em dezembro de 1996, o (CEME) tem como principal objetivo a recuperação, e preservação de fontes documentais escritas, audiovisuais, iconográficas e tridimensionais. Entendido como um lugar da memória (NORA, 2004), é também um espaço de produção cultural cuja política de documentação e informação está voltada, prioritariamente para o desenvolvimento de estratégias de acessibilidade às suas mais diversas coleções. Nessa direção, tem realizado uma gama diversa de atividades tais como exposições, mostras fotográficas, oficinas temáticas, palestras, cursos, seminários bem como a produção de pesquisas cujos resultados são publicados no formato de livros, capítulos, teses, dissertações, monografias e artigos científicos ${ }^{5}$.

O tema do lazer compõe uma das suas nove coleções específicas. ${ }^{6}$ Denominada Lazer e Recreação Pública, foi organizada a partir da doação feita pela da família do professor Frederico Guilherme Gaelzer $^{7}$ de um expressivo material referente ao desenvolvimento deste campo no Rio Grande do Sul e Brasil. Para além destes itens, a coleção apresenta vários documentos, alguns dos quais relacionados à programas sociais de esporte e lazer na qual destaca-se o acervo do Programa Segundo Tempo, seja pela sua especificidade, seja pela diversidade de registros que abriga.

Instituído pelo Ministério do Esporte em outubro de 2003 como o objetivo de "democratizar o acesso à prática e à cultura do esporte como instrumento educacional, visando o desenvolvimento integral de crianças, adolescentes e jovens, como meio de formação da cidadania e melhoria da qualidade de vida" (FILGUEIRA, 2008, p. 13-14), o Programa Segundo Tempo (PST) é gerenciado pela Secretaria Nacional de Esporte Educacional. Inicialmente voltado para atividades desenvolvidas em espaços comunitários, o Programa ampliou seu eixo de intervenção e, desde 2010, atua no contexto da educação escolar por meio de uma parceria com o Ministério da Educação, mais especificamente junto ao Programa Mais Educação. ${ }^{8}$ Em função de sua

5 Maiores informações sobre as atividades desenvolvidas pelo CEME estão disponíveis em http://www.esef.ufrgs. br/ceme/

6 O acervo do CEME está catalogado a partir de nove coleções, a saber: Escola de Educação Física; Olímpica; Educação Física e Esportes; Dança; Lazer e Recreação Pública; Movimento de Estudantes de Educação Física; Programa Segundo Tempo; Universíade 1963; Colégio Brasileiro de Ciências do Esporte. Maiores informações sobre cada coleção podem ser acessadas em http://www.esef.ufrgs.br/ceme/acervo.php

7 Sobre a atuação de Frederico Guilherme Gaelzer no campo do lazer ler: Silvia Amaral (2001), e Elza Peixoto (2007), Eneida Feix e Silvana Goellner (2008).

8 Maiores informações sobre o Programa Mais Educação podem ser acessadas em < < http://portal.mec.gov.br/ index.php? Itemid $=86 \&$ id $=12372$ \&option $=$ com content $\&$ view $=$ article $>>$ 
abrangência é considerado como um dos programas sociais com maior visibilidade no cenário das políticas públicas de esporte e lazer desenvolvidos no Brasil.

Em outubro de 2009, o Programa Segundo Tempo propôs ao Centro de Memória do Esporte uma parceria com o objetivo de construir registros sobre a sua memória gerando informações de diferente natureza: acadêmicas, históricas e de divulgação. Desde então, uma série de iniciativas foram postas em ação como, por exemplo, a criação da coleção específica do Programa Segundo Tempo no acervo do CEME com a catalogação e guarda de registros específicos; a realização de exposições temáticas, itinerantes e virtuais; a coleta de depoimentos; a criação de tutoriais que auxiliam os núcleos do Programa a registrar suas próprias memórias, a criação de uma página específica na Internet ${ }^{9} \mathrm{e}$, em julho de 2011, a criação do seu repositório digital.

Essas iniciativas estão fundamentadas na política de documentação e informação do Centro de Memória do Esporte cujas atividades estão direcionadas para a potencialização do uso de sistemas e ferramentas relacionados ao movimento de acesso livre à informação. Nesse sentido, a recente criação do Repositório Digital do Centro de Memória do Esporte ${ }^{10}$ marca nova etapa na sua atuação política e pedagógica do CEME qual seja, "construir, guardar e preservar seus acervos e, a partir deles, fomentar projetos educacionais e culturais disponibilizando-os para um infinito número de pessoas" (GOELLNER, 2003, p. 202).

\section{O REPOSITÓRIO DIGITAL DO CENTRO DE MEMÓRIA DO ESPORTE E O ACERVO MEMÓRIA DO PROGRAMA SEGUNDO TEMPO}

Atenta às políticas de produção e acesso à informação, o Centro de Memória do Esporte reuniu esforços no sentido de promover maior visibilidade e acessibilidade ao seu acervo utilizando, para tanto, ferramentas relacionadas ao acesso livre à informação por meio da criação do seu repositório digital seguindo a mesma estruturação do LUME Repositório Digital da Universidade Federal do Rio Grande do Sul ${ }^{11}$.

Essa iniciativa integra também o plano político da UFRGS dada a importância que a instituição atribui à produção do conhecimento e a sua difusão.

A tecnologia da informação tem um papel estratégico na UFRGS, não apenas como possibilidade de expansão dos serviços bibliotecários e instrumento fundamental para o ensino presencial e a distância, mas também como fonte de indicadores gerenciais e de integração com outros repositórios de ensino e pesquisa no país. A grande quantidade de conhecimento produzido nas universidades faz com que seja necessário, além da sua difusão e uso, a sua preservação, por isso a importância de projetos como este. (PAVÃO et al, 2008, p. 2).

9 Disponível em http://www.esef.ufrgs.br/ceme/pst/

10 Disponível em http://www.lume.ufrgs.br/handle/10183/40502

11 O LUME é reconhecido internacionalmente, ocupando o $41^{\circ}$ lugar dentre mais de 1.500 repositórios digitais em todo o mundo segundo o Ranking Web of World Repositories, elaborado pelo Cybermetrics Lab, grupo de pesquisa do Consejo Superior de Investigaciones Científicas (CSIC) ligado ao Ministério da Educação da Espanha. Pode ser acessado no seguinte endereço: < http://www.lume.ufrgs.br/ $>$. 
A criação de repositórios digitais tem marcado a política institucional de várias outras universidades brasileiras com a função de compartilhar o que é produzido pelos seus pesquisadores. Segundo Masson (2008) os repositórios apresentam algumas características específicas, a saber: a) são tecnologias de informação desenvolvidas para organizar, coletar, disseminar e preservar informações e conhecimentos; b) são ferramentas criadas para contribuir com o avanço de pesquisas; c) sua arquitetura tem forma de rede interoperáveis possibilitando novas formas de avaliação das produções científicas e do desempenho dos pesquisadores bem como a interação e democratização do acesso à informação e conhecimento potencializando a colaboração entre pesquisadores e a sociedade em geral; d) são ferramentas flexíveis que podem ser adaptadas às constantes e rápidas mudanças da sociedade contemporânea; e) são sistemas que surgem no contexto da convergência tecnológica digital tendo na Internet o seu ponto central.

Para o desenvolvimento dos repositórios digitais foi importante a implementação do software DSpace, recomendado pelo Instituto Brasileiro de Informação em Ciência e Tecnologia (IBCIT) por possibilitar a criação destes repositórios

com funções de captura, distribuição e preservação da produção intelectual, permitindo sua adoção por outras instituições em forma consorciada federada.
O sistema desde seu início teve a característica de ser facilmente adaptado a outras instituições. Os repositórios DSpace permitem o gerenciamento da produção científica em qualquer tipo de material digital, dando-lhe maior visibilidade e garantindo a sua acessibilidade ao longo do tempo (INSTITUTO BRASILEIRO DE INFORMAÇÃO EM CIÊNCIA E TECNOLOGIA, 2012).

Considerando a experiência do LUME com o DSpace e a atuação da Biblioteca Edgard Sperb (ESEF/UFRGS) com a plataforma Sistema Eletrônica de Editoração de Revistas (SEER) ${ }^{12}$, o Centro de Memória do Esporte aderiu ao movimento do acesso livre à informação criando o seu repositório específico $^{13}$ (iniciativa pioneira na UFRGS) que, segundo Kuramoto (2008) pode ser classificado como temático visto que nele são depositados apenas documentos de uma determinada área do conhecimento.

O Repositório Digital do Centro de Memória do Esporte foi incorporado ao LUME em maio de 2012 e compõe uma de suas sub-comunidades. Está estruturado a partir de dois acervos denominados de Centro de Memória do Esporte e Memória do Programa Segundo Tempo, cada qual contendo cinco coleções: Audiovisual, Depoimentos, Documental, Iconográfica e Tridimensional

Vale destacar que a criação do Repositório foi inspirada também no Repositório Institucional da Rede Cedes (RIRC) ${ }^{14}$ implementado pelo LaboMídia - Laboratório

12 A Biblioteca compõe a equipe editorial e administrativa da Revista Movimento sendo responsável pela sua publicação no SEER desde 2007.

13 Essa iniciativa foi potencializada pela aquisição de um servidor específico alocado junto ao Centro de Processamento de Dados da UFRGS, com financiamento do Ministério do Esporte através do Projeto Memória do Programa Segundo Tempo.

14 Disponível em http://www.labomidia.ufsc.br/redecedes/ 
e Grupo de Pesquisa em Mídia Esportiva ${ }^{15}$ em parceira com o Ministério do Esporte, mais especificamente, a Secretaria Nacional de Desenvolvimento do Esporte e Lazer. Os objetivos do RIRC são assim explicitados:

reunir, preservar, organizar e disponibilizar produtos resultantes das pesquisas acadêmicas apoiadas pela Rede CEDES; contribuir para dar visibilidade à produção da Rede CEDES; permitir o acesso através da Internet à produção da Rede CEDES; preservar a memória do trabalho acadêmicos científico da Rede CEDES; participar ativamente, enquanto parceiro, no esforço conjunto da comunidade científica nacional e internacional, no movimento de acesso livre à informação acadêmica (CENTRO DE DESENVOLVIMENTO DO ESPORTE RECREATIVO E LAZER, 2012).

A experiência já consolidada do Repositório Institucional da Rede Cedes, em que pese as dificuldades relativas à participação da comunidade que integra a Rede no que respeita ao auto-arquivamento ${ }^{16}$, indica a relevância desses suportes para a preservação da memória de programas sociais em esporte e lazer. Segundo Ferrari,

os estudos do eixo memória do esporte e lazer da Rede CEDES representam quase um quinto das pesquisas $(19,58 \%)$, grande parte delas relacionadas a levantamento de fontes para construção de centros de memória. Diante desses dados, compreendemos que a melhor utilização do RIRC e a ampliação dos repositórios digitais facilitaria a realização de pesquisas nessa perspectiva no futuro. Aproveitamos essa reflexão para finalizar este texto, realçando a relevância atual do auto-arquivamento no âmbito da gestão da informação e do conhecimento relacionada com o nosso presente, porque no futuro, serão essas produções que possibilitarão as pesquisas sobre a memória do esporte e lazer no Brasil. (FERRARI, 2012, p. 156).

Levando em consideração a intenção política e pedagógica de preservar a memória Programa Segundo Tempo criamos um acervo específico dentro do Repositório Digital do Centro de Memória do Esporte cujos itens estão catalogados a partir de cinco coleções: a) Audiovisual - abriga documentos no formato de vídeos (filmes, depoimentos, slides, videoconferências, etc.) e sonoros (entrevistas de rádio, jingles, músicas, etc.); b) Depoimentos - comporta as entrevistas realizadas pelo projeto $\mathrm{Ga}$ rimpando Memórias. ${ }^{17}$ Essas entrevistas são disponibilizadas na íntegra para consulta, tanto no Repositório quanto no Sistema Automatizado das Bibliotecas (SABi), o catálogo eletrônico do Sistema de Bibliotecas da UFRGS ${ }^{18}$; c) Documentos - caracterizada por documentos de diferente natureza tais como livros, periódicos, leis, atas, reportagens de jornais e revistas, correspondências, apresentações em Power Point, entre tantas outras. Para a sua disponibilização

15 Vinculado à Universidade federal de Santa Catarina. http://www.labomidia.ufsc.br/

16 Sobre esse tema ler a dissertação de Rodrigo Ferrari, Gestão da informação e conhecimento em esporte e lazer: o caso do Repositório Institucional da Rede Cedes (RIRC),

17 Desenvolvido pelo CEME, objetiva preservar e divulgar a memória das práticas corporais e esportivas no Brasil. Fundamenta-se no aporte teórico-metodológico da História Cultural e da História Oral e tem como objetivo a realização de entrevistas e sua transformação em documento escrito que pode servir de fonte para quem se interessar por pesquisas afins aos temas desenvolvidos no projeto. Maiores informações podem ser acessadas em http://www.esef.ufrgs.br/ceme/projPesq.php?acao = ver\&id $=1$ 
no Repositório estes documentos foram digitalizados e publicados em formato pdf. Destacamos todos os livros publicados pelo Programa que podem ser acessados tanto nesta coleção como na exposição virtual A Produção do Programa Segundo Tempo: livros e cadernos pedagógicos, veiculada na página que o Projeto Memória do Programa Segundo Tempo mantém na Internet ${ }^{19}$; d) Iconográfica - comporta imagens em diferente suportes: fotografias, cartazes, desenhos, pinturas, banners, entre outras. Além desse material, é possível visualizar as exposições presenciais, itinerantes e virtuais realizadas com temáticas relacionadas ao Programa. Destacamos que a criação de exposições virtuais tem como objetivo a divulgação do acervo do Programa a partir de recortes temáticos. Além da exposição já mencionada sobre a produção de livros e cadernos pedagógicos, estão também disponíveis para visitação as exposições Memórias do Programa Segundo Tempo, Recreio nas Férias ( $1^{\text {a }}$ edição) e Projetos Especiais $^{20}$; e) Tridimensional - abriga objetos tais como vestuário, medalhas, objetos e equipamentos esportivos, canetas, pastas, entre outros. Esses objetos são fotografados e as imagens podem ser visualizadas no Repositório acompanhadas de suas especificidades como, por exemplo, tamanho, peso, textura, bem como a origem e a história de cada peça, ressaltando, se já integrou alguma exposição, seja ela organizada pelo próprio CEME ou emprestada para outra instituição.

Essas coleções podem ser pesquisadas através de metadados (dados que descrevem o documento) criados especificamente para facilitar as buscas e acessos, a saber: ano, autor, título, palavras-chave e acervos. A recuperação baseia-se nas pesquisas de modo simples e avançado, percorrendo as coleções e acervos e o sistema oferece, caso haja interesse em conhecer, as estatística de uso dos itens, indica os mais acessados bem como os países e as cidades que protagonizam os acessos.

Acreditamos, ainda, que outro aspecto inovador deste Repositório consiste no acesso à diversidade dos registros que integram cada coleção e que podem ser facilmente utilizados pelo usuário. Além de visualizar o documento e coletar informações específicas sobre ele, é possível fazer um download e, assim, utilizá-lo em suas pesquisas e interesses.

Registramos, ainda, que à semeIhança do Repositório Institucional da Rede Cedes, o acervo Memória do Programa Segundo Tempo do Repositório Digital do Centro de Memória do Esporte opera a partir de lógica próprias. Ou seja, ainda que financiada pelo Ministério do Esporte via um programa social de esporte e lazer, sua manutenção se dá de modo relativamente independente dele. Ferrari (2012), ao analisar os repositórios da Rede CEDES e do CEME afirma que essas iniciativas

são apoiadas e financiadas pelo ministério a despeito de uma organização interna que considere a gestão da informação e do conhecimento como política pública útil e necessária. Se esses repositórios deixassem de ser propostas marginais no contexto da administração ministerial,

19 A exposição pode ser acessada em http://www.esef.ufrgs.br/ceme/pst/exposicaoVirtual.php?exposicao = 4

20 Disponíveis em http://www.esef.ufrgs.br/ceme/pst/exposicoesVirtuais.php 
eles deveriam ser uma das estruturas de uma organização que envolva todo o ME. Os repositórios são ferramentas muito eficientes e indispensáveis em projetos democráticos de governança, com isso, o ME pode se tornar um exemplo a ser seguido por outros ministérios brasileiros caso seja pioneiro na organização de uma proposta de gestão da informação e conhecimento que envolva todo ME (p. 146-147).

\section{CONSIDERAÇÕES FINAIS}

O acervo Memória do Programa Segundo Tempo encontra-se, ainda, em sua fase inicial. Os documentos disponibilizados resultam de um primeiro esforço no que respeita ao seu mapeamento, digitalização, conversão em formatos compatíveis para que possam ser acessados e sobretudo, a produção de novos registros.

Em que pese esse caráter embrionário, entendemos que a criação e manutenção da comunidade Memória do Programa Segundo Tempo representa um avanço para a política de gestão da informação na área dos programas sociais de esporte e lazer. A divulgação de seu acervo digitalizado, preservado em seus originais e difundido pelo sistema eletrônico trabalha com a "busca do sentido, oferecendo a possibilidade de, a partir de correlações que se estabelecem na construção da informação, apresentar o objeto em seus diferentes contextos e sugerir possibilidades de apropriação e de participação efetiva dos públicos de modo presencial e virtual" (LARA FILHO, 2009, p. 168).

Sua continuidade pressupõe também a implementação de estratégias que envolvem um número significativo de pessoas. Além das equipes CEME, do Centro de Processamento de Dados da UFRGS e do Ministério do Esporte demanda esforços no sentido de sensibilizar gestores, coordenadores de equipes e núcleos, professores, assessores, monitores e participantes do Programa para importância do registro daquilo que realizam cotidianamente nas diferentes áreas de intervenção do Programa. Implica, sobretudo, explicitar que a memória não nos aprisiona ao passado mas conduz à indagar o presente para melhor compreendê-lo. A memória, portanto, é matéria viva e terreno fértil para a produção de diferentes histórias. Neste caso específico, a produção de histórias acerca de um programa social de esporte e lazer que tem marcado o cenário das políticas públicas brasileiras e sobre o qual se pode fazer várias leituras e interpretações.

\section{REFERÊNCIAS}

AMARAL, Silvia C. F. Espaços e vivências públicas de lazer em porto alegre: da consolidação da ordem burguesa à busca da modernidade urbana. Revista Brasileira de Ciências do Esporte, vol. 23, n. 1, p. 109-121, /2001.

CONSELHO NACIONAL DE ARQUIVOS. Recomendações para digitalização de documentos arquivísticos permanentes. abr. 2010. Disponível em: <www. conarq.arquivonacional.gov.br/.../ recomendaes_para_digitalizacao $>$. Acesso em: 12 abr. 2012.

CENTRO DE DESENVOLVIMENTO DO ESPORTE RECREATIVO E LAZER. Política do Repositório Institucional Rede CEDES. Disponível em: < http:// www.labomidia.ufsc.br/redecedes/ politica.html>. Acesso em: 21 maio 2012.

FEIX, Eneida; GOELLNER, Silvana V. O florescimento dos espaços públicos de 
lazer e de recreação em Porto Alegre e o protagonismo de Frederico Guilherme Gaelzer. Licere, 2008, vol. 13, p. 1-18, 2008.

FERRARI, Rodrigo D. Gestão da informação e conhecimento em esporte e lazer: o caso do Repositório Institucional da Rede Cedes (RIRC). 2012. Dissertação (Mestrado) - Programa de Pós-Graduação em Educação Física, Universidade Federal de Santa Catarina, Florianópolis, 2012.

FILGUEIRA, Júlio. Gestão de projetos esportivos sociais. In: OLIVEIRA, Amauri B. de; PERIM, Gianna L. (org.) Fundamentos Pedagógicos para o Programa Segundo Tempo: $1^{\circ}$ Ciclo Nacional de Capacitação dos Coordenadores de Núcleo. Brasília: Ministério do Esporte; Porto Alegre: UFRGS, 2008.

GOELLNER, Silvana V. Informação e documentação em esporte, educação física e lazer: o papel pedagógico do Centro de Memória do Esporte. Revista Brasileira de Ciências do Esporte, Florianópolis, v. 25, n. 1, p. 199-207, 2003.

INSTITUTO BRASILEIRO DE INFORMAÇÃO EM CIÊNCIA E TECNOLOGIA DSpace: repositórios digitais. Disponível em: $<$ http://dspace.ibict.br/>. Acesso em: 28 abr. 2012.

KURAMOTO, H. Disitnguindo conceitos de repositórios e publicações eletrônicas. Blog do Kuramoto, 8 dez. 2008. Disponivel em: <http://kuramoto. blog.br/2008/12/08/distinguindoos-conceitos-de-repositorios-epublicacoes-eletronicas/ $>$. Acesso em: 12 abr. 2011.

LARA FILHO, Durval de. Museu, objeto e informação TransInformação,
Campinas, v. 21, n. 2, p. 163-169, maio/ago. 2009.

LEITE, Fernando César L.; MORENO, Fernanda P.M.; MÁRDERO ARELLANO, Miguel A. Acesso livre a publicações e repositórios digitais em ciência da informação no Brasil. Perspectivas em Ciência da Informação, Belo Horizonte, v. 11, n. 1, p. 82-94, jan./abr. 2006.

MASSON, Sílvia M. Os repositórios digitais no âmbito da Sociedade Informacional.

PRISMA.COM, n. 7, 2008.

NORA, Pierre. Entre memóire et histoire: la problématique des lieux. In: NORA, P. (Org.). Les lieux de mémoire. Paris: Gallimard, 1984. p. VII-XLII.

PAVÃO, Caterina G. et al. Repositório digital: acesso livre à informação na Universidade Federal do Rio Grande do Sul. In: SEMINARIO NACIONAL DE BIBLIOTECAS UNIVERSITARIAS, 15. SÃO PAULO, 2008. Anais... 1CD. PEIXOTO, Elza. Levantamento do estado da arte nos estudos do lazer: (Brasil) séculos XX e XXI - alguns apontamentos. Educação e Sociedade, vol. 29, n. 99, p. 561-586, 2007.

UNIVERSIDADE FEDERAL DO RIO GRANDE DO SUL. LUME: Repositório Digital da Universidade Federal do Rio Grande do Sul. Disponível em: < http:// www.lume.ufrgs.br/> . Acesso em: mai. 2012. 
Ano XXIV, n 38 , junho/2012

MEMORY AND SOCIAL PROGRAMS OF SPORT AND LEISURE: the inventory of the 'Second-Half' Program of the Digital Repository of the Center of Sport Memory

\begin{abstract}
Regarding the importance of production and spread of knowledge, this text aims at presenting some activities developed by the Center of Sport Memory of the School of Physical Education of the Federal University of Rio Grande do Sul (CEME) concerning the preservation of sport and leisure memory. The study focuses on the recent creation of the institutional repository that shelters the inventory Memory of the 'Second-Half' Program, which aims at designing strategies to publicize the production of this social program of sport and leisure in order to preserve their memory and spread it in the community as a whole.
\end{abstract}

Keywords: memory; sport; social program.

Recebido em: março/2012

Aprovado em: junho/2012 